\title{
Aspect hydrométéorologique des crues cévenoles des 30 septembre et 4 octobre 1958
}

\author{
The hydro-meteorological aspects \\ of the floods in the Cevennes \\ on September 30th and October 4th 1958
}

\author{
PAR P. GUILILO'T
}

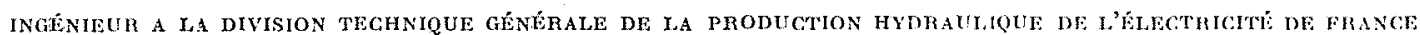

\begin{abstract}
Les pluies des 29-30 septembre 1958 , qui ont été abondantes sur toute la France, ont atteinl sur le versant sud-est des Cévennes, dans le milien de la journée du 30 septembre, une intensité exceptionnelle. Les crues subites des conrs d'eau, d'autant plus surprenantes pour les riverains que les eaux sont passes, en quelques heures, de leur niveau d'étiage à des cotes dépassant par endroits la dizaine de mètres, ont entrainé, spécialement dans le bassin da Gard, le soir du 30 , les pertes de vies humaines et les dégats matériels que l'on sait. De nouvelles précipitations survenant quatre jours plus tard ont pul faire craindre une seconde catastrophe. Sans revêtir partout un caractère aussi grave que la crue du 30 septembre, les débits constatés le 4 octobre ont atteint, en certains points, des valeurs spécifiques particulierement élevées (qu'il convient toutefois, dans plusieur's cas, d'accepter abec réserve).

La présente note se propose de donner une analyse du phenomine a partir des observations faites dans le résea exploité par les Centres Hydrométéorologiques d'E.D.F. et d'en tirer quelques conclusions pratiques.
\end{abstract}

The rain which fell on Seplember 29 th and 30 th 1958, was heavy all over France but becane exceptionally intense on the south-west slopes of the Cevennes around midday on September 30th. Rivers in the district took inhabitants by surprise and rose from low luater level to depths of many metres in a few hours, causing deaths and damage, especially in the Gard basin on the evening of September $30 t h$.

A furlher catastrophe might have been expected as a result of fresh rainfall which took place four days later. Although not as severe as on September $30 t h$, it caused particularly high specific discharges on October 4th. Some of these figures have nevertheless to be treated with caution.

This article analyses the floods on the basis of data furnished by the E.D.F. river and rain gatuge network and draws practical conclusions from this analysis.

\section{I. - PRECIPITATIONS}

La carte $\mathrm{n}^{\circ} 1$ indique la hauteur des précipilations tombées au cours des journées des 29 et 30 septembre 1958 : on voit, cu'autour du maximum de $429 \mathrm{~mm}$ relevé à Saint-EtienneVallée-Française dans le bassin du Gard, la précipitation a dépassé $300 \mathrm{~mm}$ sur une zone longue 


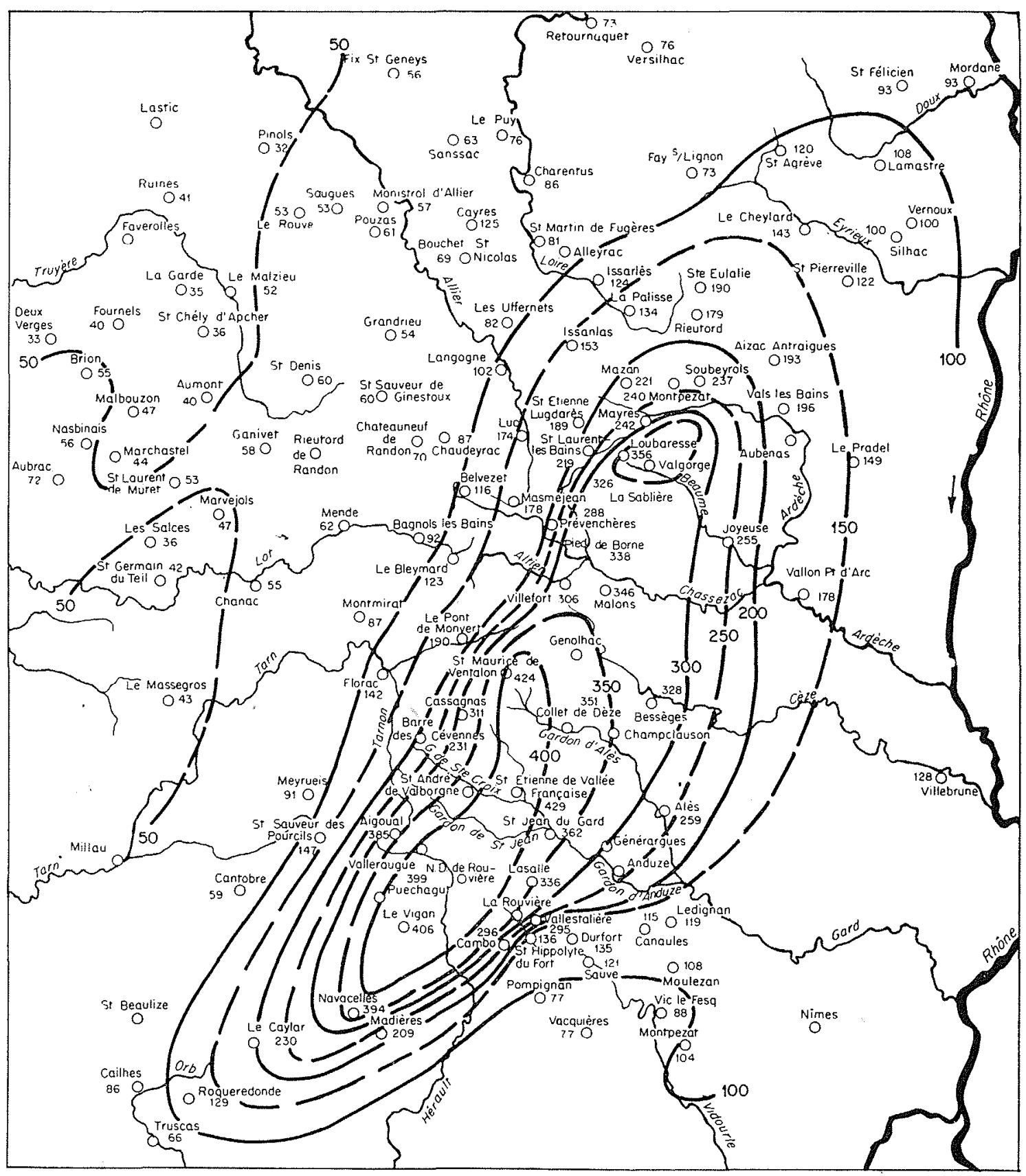

FIG. 1

Précipitations du 29-9 à $8 \mathrm{~h}$ au $1-10-58$ à $8 \mathrm{~h}$

de $100 \mathrm{~km}$ et large de 30 qui couvre les contreforts sud-est de toute la chaine des Cévennes. Sur la carte $\mathrm{n}^{\prime 2}$, figurent les isohyètes du 4 octobre. On trouve, dans les deux cas, une dorsale dont l'axe est parallèle à la ligne NNE-SSW Mézenc-Aigoual, passant sensiblement par Montpezat, le confluent Borne-Chassezac, St-EtienneVallée Française et Le Vigan. Cependant, le 4 octobre, une deuxième dorsale parallèle appa- raît dans la moitié sud, à 25 à $30 \mathrm{~km}$ à l'est de la première, sur Pompignan-Alès.

En quelques points, la valeur indiquée est une estimation par défaut, le pluviomètre, dont la capacité est d'environ $180 \mathrm{~mm}$, ayant débordé avant que l'observateur ait fait une mesure intermédiaire. Il n'y a pas lieu cependant de généraliser ni d'exagérer l'importance des erreurs résultants des débordements des pluviomètres. 


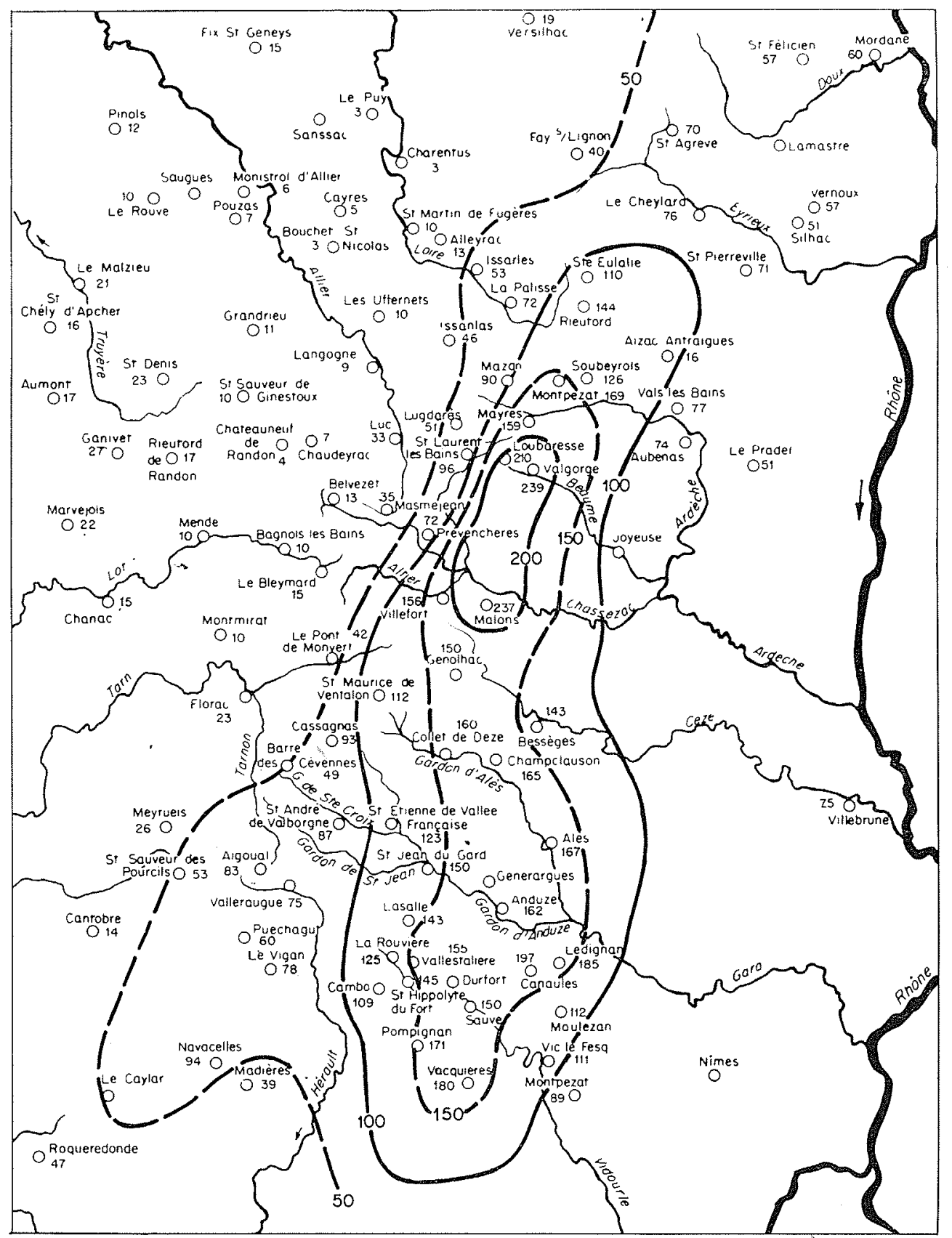

FiG. 2

Précipitations du 3-10 à 8 h au 5-10-58 à $8 \mathrm{~h}$

A part quelques cas qui s'éliminent d'eux-mèmes par le tracé des isohyètes, la plupart des observateurs sont dignes de confiance, et ont fait des mesures intermédiaires quand cela a été nécessaire. Certains étaient déjà pourvus par E.D.F., et tous l'ont été au cours des derniers mois dans les Cévennes et les Pyrénées orientales, de seaux "modèle profond» qui contiennent 280 et non $180 \mathrm{~mm}$.
Les douze pluviographes (1) installés dans cette région par E.D.F. en liaison, dans la partie la plus méridionale, avec la C.N.B.R.L., ont fourni des indications précieuses sur la chronologie du phénomène (voir les pluviogrammes sur le

(1) 5 nouveaux appareils viennent d'être installés par E.D.F. en 1959, dont 2 dans le bassin du Gard et 3 dans celui de l'Ardèche. 


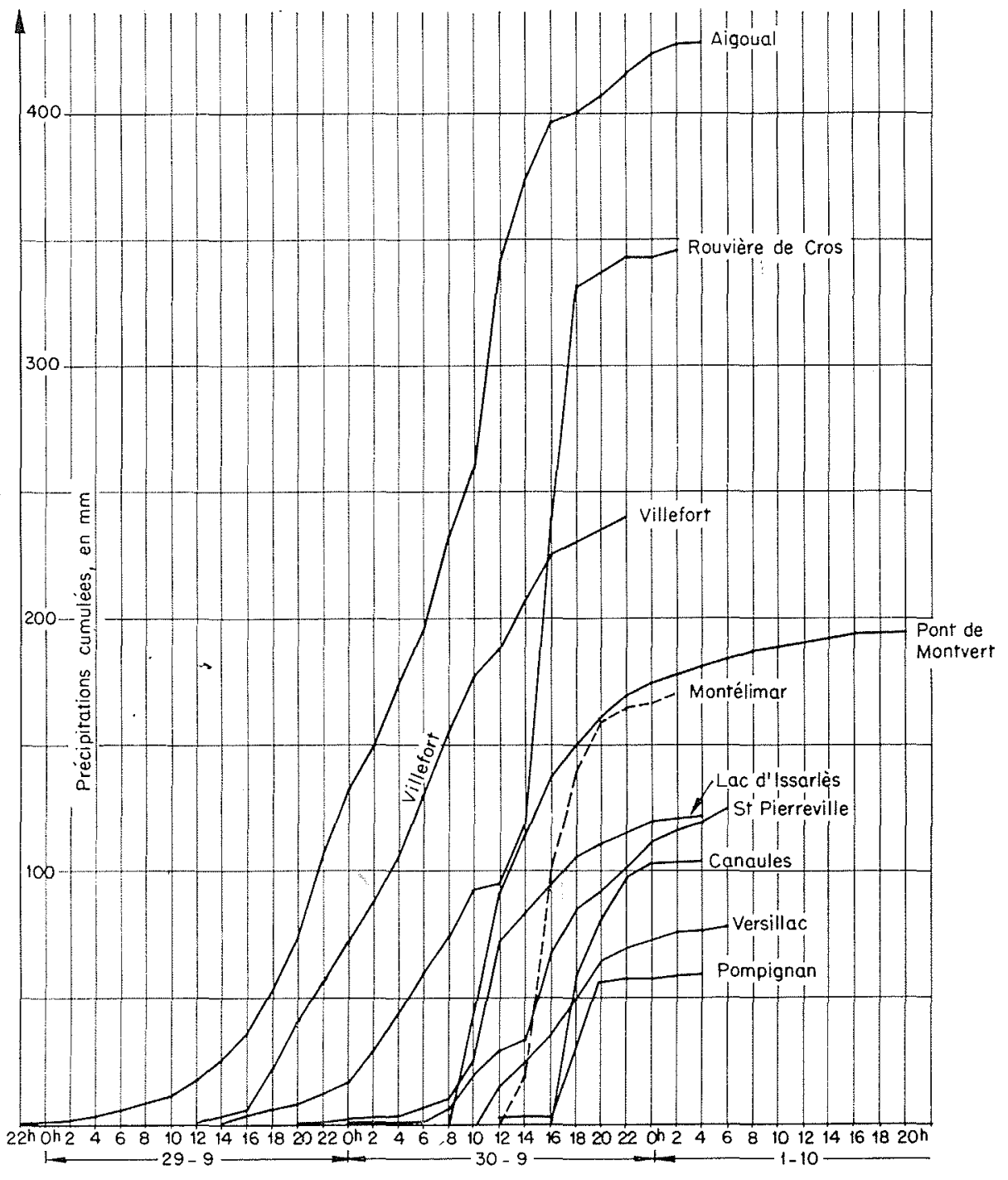

Fig. 3

Pluviogrammes du 29-9 au 1-10-58

plan $\mathrm{n}^{\circ} 3$ ). C'est bien l'averse du 30 septembre, $210 \mathrm{~mm}$ entre 13 et 17 heures à la Rouvière, qui paraît la cause directe de la crue catastrophique. L'examen des pluviogrammes du 28 au 30 septembre 1958 montre la propagation d'ouest en est, au cours de la journée du 30 , de l'averse violente liée à l'arrivée de l'air froid, propagation qui apparait sur la carte $n^{\circ} 4$ représentant les isochrones du début de l'averse. Cette recrudescence est relativement plus sensible à basse altitude que sur l'alignement des crêtes; car, dans les secteurs privilégiés où l'effet d'accrochage par le relief cévenol est le plus intense (versant sud-est du Mézenc, du Tanargue, du mont Lozère et de l'Aigoual), l'averse de front froid est habituellement précédée d'une pluie continue due au soulèvement par les Cévennes de l'air chaud et humide soufflant du sud. Cette pluie purement orographique avait débuté dès le 29 septembre à $14 \mathrm{~h}$ à la Rouvière, à $12 \mathrm{~h}$ à Villefort, le $28 \dot{a} 22 \mathrm{~h}$ a l'Aigoual, et y avait déjà apporté respectivement, 100,150 et $250 \mathrm{~mm}$. C'est pourquoi on peut dire que l'Aigoual, à l'avant-garde sud des Cévennes, est le bon «témoin».

Nous donnons plus loin une analyse plus détaillée de l'évolution météorologique au cours de ces journées et du deuxième épisode qui suivit.

Le 4 octobre, le déroulement a été plus rapide, puisque tout s'est passé dans les 12 premières heures de la journée, avec le maximum d'intensité passant entre 4 et $6 \mathrm{~h}$ sur la limite ouest (ligne Aigoual, Villefort, Montpezat), entre 8 et $10 \mathrm{~h}$ sur la limite est (Pompignan-Alès). 


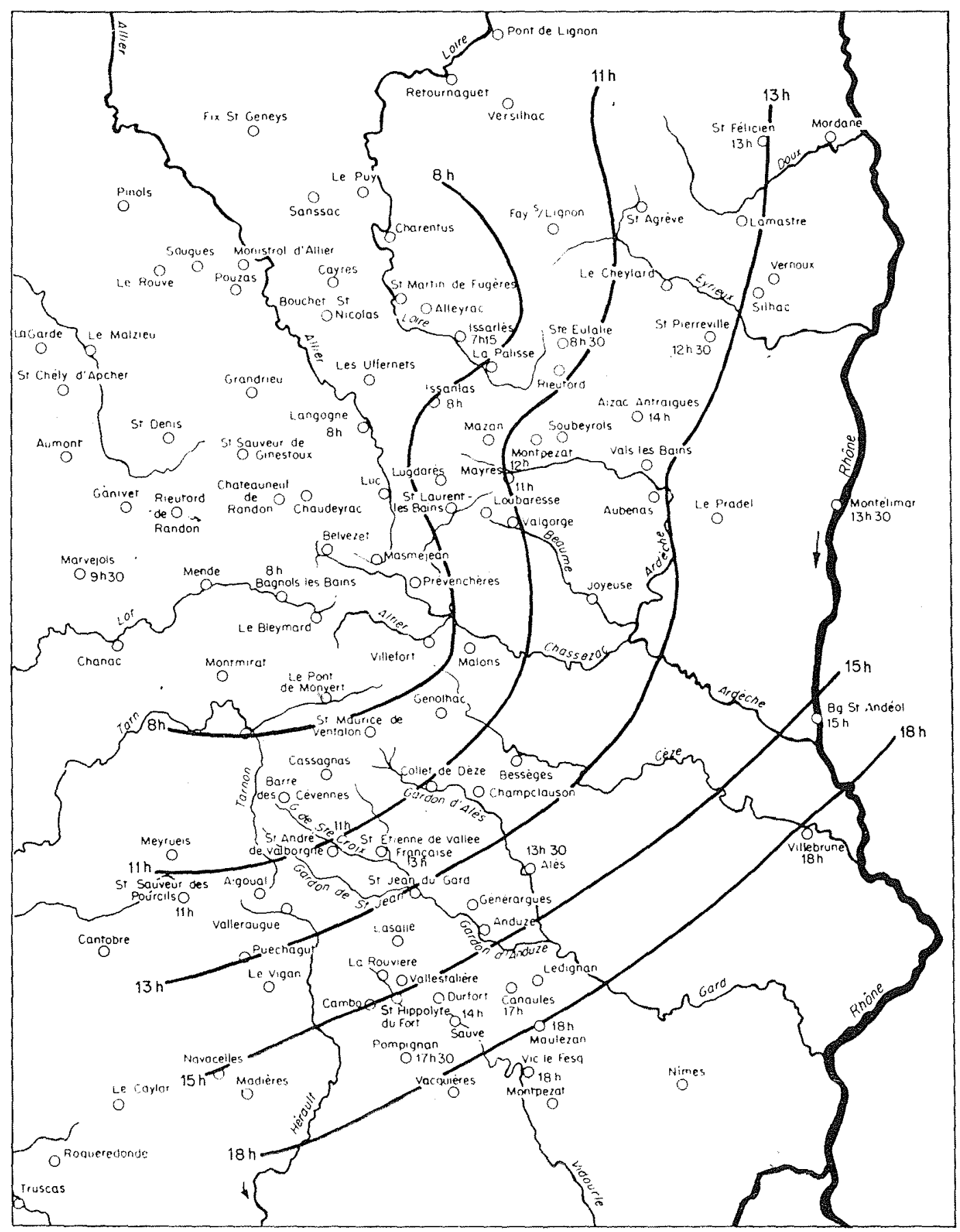

Fig. 4

Heure du début de l'averse torrentielle du 30 septembre 1958

\section{II. - DËBITS}

On a rassemblé dans le tableau de la figure 5, pour un certain nombre de points de mesure caractéristiques des différents bassins, les ren- seignements sur l'horaire des crues et les valeurs maximales des niveaux ou des débits. Beaucoup de ces valeurs de débits résultent mal- 


\begin{tabular}{|c|c|c|c|c|c|c|c|c|c|}
\hline \multirow{3}{*}{ POINT DE MESURE } & \multirow{3}{*}{$\begin{array}{c}B . V . \\
\text { cn } \mathrm{km}^{2}\end{array}$} & \multicolumn{4}{|c|}{ Crus du $30-9-1958$} & \multicolumn{4}{|c|}{ crues du 4-10-1958 } \\
\hline & & \multirow{2}{*}{\multicolumn{2}{|c|}{$\begin{array}{l}\text { Heure et cote } \\
\text { du maximum }\end{array}$}} & \multicolumn{2}{|c|}{ Débit } & \multirow{2}{*}{\multicolumn{2}{|c|}{$\begin{array}{l}\text { Heure et cote } \\
\text { du maximum }\end{array}$}} & \multicolumn{2}{|c|}{ Débit } \\
\hline & & & & $\mathrm{m}^{3 / \mathrm{s}}$ & $1 / \mathrm{s} / \mathrm{km}^{2}$ & & & $\mathrm{~m}^{3 / 3} \mathrm{~s}$ & $1 / \mathrm{s} / \mathrm{km}$ \\
\hline L'Eyrieux au pont de Chervil... & 392 & $15 \mathrm{ln} 30$ & 3,55 & 160 & 400 & $7 \mathrm{~h} 30$ & 4,30 & 270 & 700 \\
\hline Le Doux à Pont-du-Verger ....... & 190 & $22 \mathrm{~h} 00$ & 1,68 & $32 ?$ & $170 ?$ & $15 \mathrm{~h} 00$ & 1,84 & 39 ? & $200 ?$ \\
\hline La Loire à Rieutort ...... & 61 & $14 \mathrm{~h} 30$ & 2,40 & 89 & 1460 & $7 \ln 30$ & 3,95 & 208 & 3400 \\
\hline La Borne aux Chambons... & 11 & $12 \mathrm{~h} 00$ & 2,70 & $100 ?$ & $9000 ?$ & $8 \mathrm{~h} 45$ & 2,66 & $90 ?$ & $8000 ?$ \\
\hline L'Altier à Castanet .... & 113 & $15 \mathrm{~h} 00$ & 2,90 & 87 & 750 & $7 \mathrm{~h} 00$ & 2,07 & 35 & 300 \\
\hline Chassezac à Albespeyre & 107 & $13 \mathrm{ho0}$ & 2,48 & 185 & 1700 & $7 \mathrm{~h} 30$ & 1,78 & 65 & 600 \\
\hline Ardèche à Vallon-Pont-D'Arc & 1840 & 20 h 00 & 12,20 & $4000 ?$ & $2300 ?$ & 14 h 00 & 10,30 & 3400 ? & $1800 ?$ \\
\hline Ardèche à Sauze-Saint-Martin & 2140 & $22 \mathrm{~h} 00$ & 9,30 & $4500 ?$ & 2100 & 15 h 30 & 8,35 & $3600 ?$ & 1700 \\
\hline Gardon de Saint-Jean à Saint-Jean-du-Gard & 160 & 14 h 15 & 2,80 & & & 9 h 30 & 0,60 & & \\
\hline Gardon d'Anduze à Anduze . . . . . . . . . & 544 & $17 \mathrm{~h} 00$ & 6,00 & & & $11 \mathrm{~h} 00$ & 5,30 & & \\
\hline 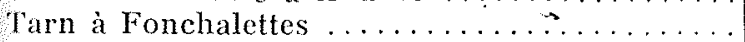 & 67 & $13 \mathrm{n} 30$ & 4,50 & 260 & 3900 & $9 \mathrm{~h} 00$ & 4,15 & 200 & 3000 \\
\hline Tarn à La Malène ................ & 818 & $16 \pitchfork 30$ & 4,13 & 1400 & 1700 & $16 \mathrm{~h} 30$ & 1,70 & 390 & 480 \\
\hline Vidourle à Saint-Hippolyte... & 35 & $16 \mathrm{~h} 30$ & 1,93 & 190 & 5400 & $9 \mathrm{~h} 00$ & 1,72 & 140 & 4000 \\
\hline Vidourle à Sommières...... & 630 & 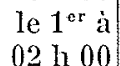 & 2,60 & 350 & 550 & $16 \mathrm{~h} 00$ & 6,76 & $1000 ?$ & $1600 ?$ \\
\hline Vis à Madières & 282 & $17 \mathrm{~h} 00$ & & $(600 ?)$ & $2000 ?$ & $10 \mathrm{~h} 00$ & & $(400 ?)$ & $1400 ?$ \\
\hline Hérault à Moulin-Bertrand & 1090 & 18 h 30 & 4,36 & $2500 ?$ & $2300 ?$ & $13 \mathrm{~h} 00$ & 2,00 & 700 & 650 \\
\hline
\end{tabular}

FIG. 5

Tableau des valeurs maximales des niveaux et des débits

heureusement d'extrapolation, et doivent être considérées comme des estimations provisoires. De nombreuses stations de mesure ont en effet été détruites ou ont fourni des résultats difficilement exploitables au cours de ces journées.

On voit néanmoins que la crue du 4 octobre a été moins grave que celle du 30 septembre au centre de la zone, dans les bassins du Gard et de l'Ardèche. Par contre, au nord, la Loire, au sud, le Vidourle, ont eu le 4 octobre une crue plus forte que le 30 septembre, soit que le sol soit resté saturé de la première averse, soit que l'intensité ait été localement plus forte soit encore - c'est le cas du Vidourle, analysé dans l'étude de M. Jacquet - que la crue ait été aggravée par une propagation coïncidente de l'averse de l'amont vers l'aval.

\section{COMMENTAIRES}

Notre intention n'est pas d'ajouler à l'abondante littérature sur les averses cévenoles, mais de dégager quelques conclusions pratiques, en particulier pour les Services d'Exploitation E.D.F.

\section{Le phénomène n'est pas rare sur les Cévennes.}

La probalité du retour d'une crue comme celle du 30 septembre 1958 sur l'une quelconque des rivières cévenoles: Hérault, Vidourle, Gard, Cìze, Ardèche, Doux, Eyrieux, n'est nullement millénaire. Plusieurs fois chaque année, le plus souvent en automne, mais parfois aussi jusqu'en mars ou avril, des aver'ses de 100 à $200 \mathrm{~mm}$ en moins de 24 heures se produisent sur cette région, et peu de conditions supplémentaires sont à réunir pour passer localement de 200 à $400 \mathrm{~mm}$. En outre, le retour est fréquent de ce type d'averse à quelques jours d'intervalle, témoin celle du 4 octobre 1958, retour qui 


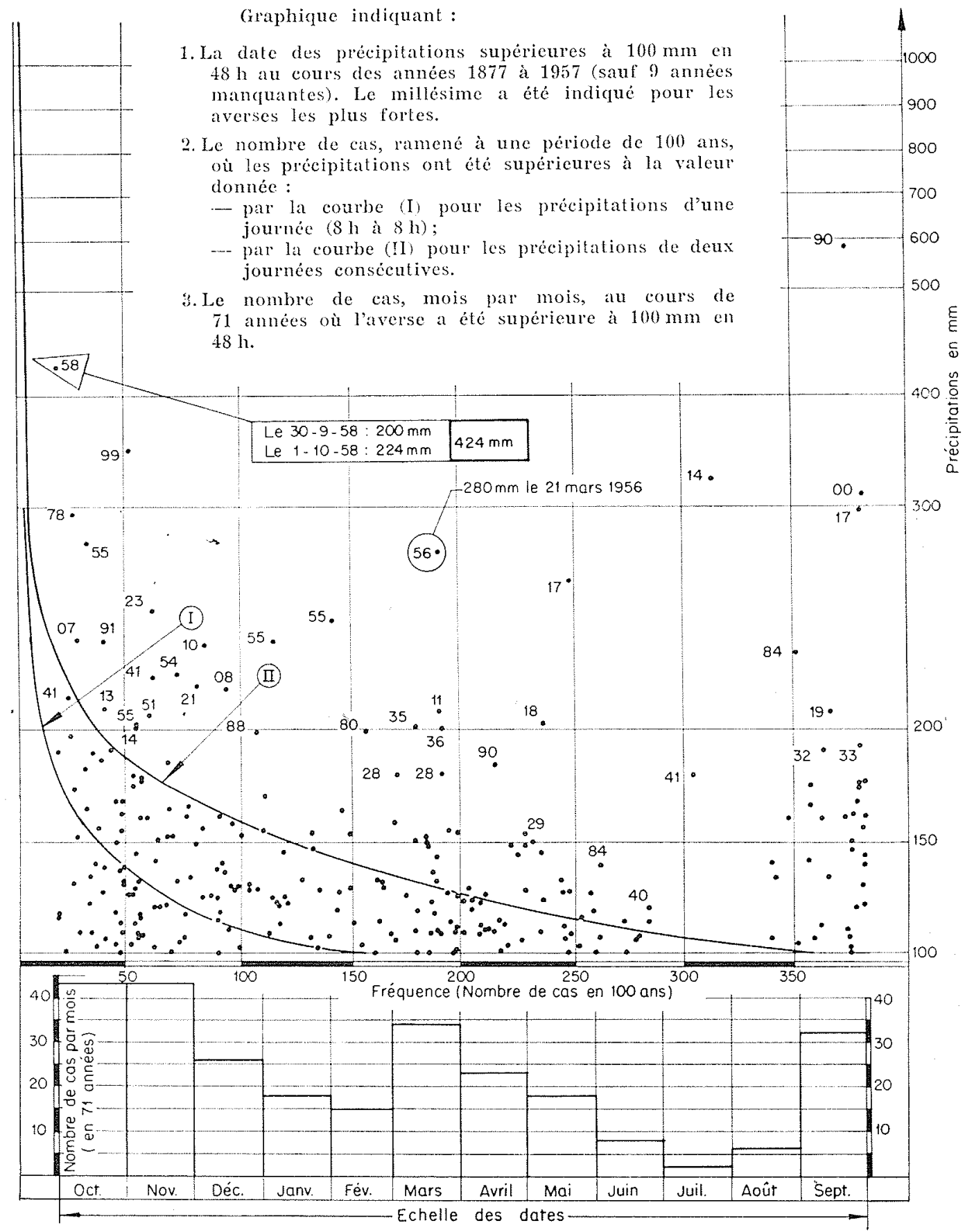

Fia. 6

Dates et fréquence des averses observées à Vialas

s'explique simplement par le passage d'une famille de perturbations ayant des trajectoires voisines.

Le dépouillement des données pluviométriques journalières que nous avons pu trouver pour trois stations : Montpezat, Vialas et Valleraugue, sur une série de 70 années, nous a permis d'encadrer la courbe de fréquence des précipitations supérieures à $100 \mathrm{~mm}$ au cours de
24 heures consécutives : on a tracé les courbes de fréquence correspondant, l'une au cas d'un relevé journalier ( $08 \mathrm{~h}$ à $08 \mathrm{~h}$ ), l'autre au cas de deux relevés quotidiens consécutifs dont le total est supérieur à $100 \mathrm{~mm}$. La figure 6 illustre le cas de Vialas. Pour autant que ces données soient valables, les précipitations de 2 journées pluviométriques $(08 \mathrm{~h}$ à $08 \mathrm{~h})$ consécutives ont été supérieures à $100 \mathrm{~mm}, 3,6$ fois par an à 


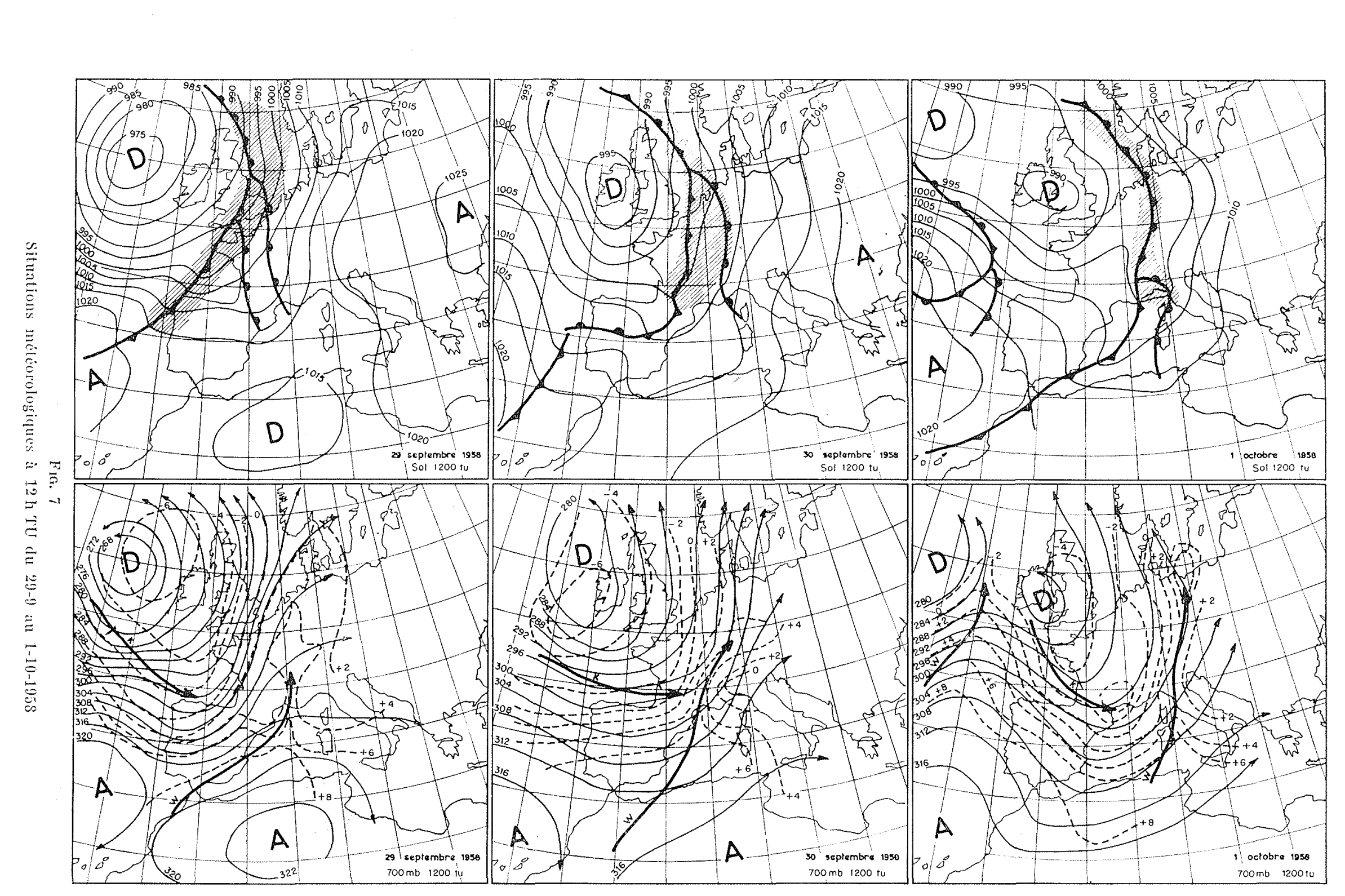


Vialas, 3,1 fois à Vallerangue, 2,6 fois à Montpezat.

Les dates et les décomptes mensuels des averses (correspondant aux relevés sur 48 h) figurent également sur ces graphiques. C'est dans le trimestre septembre à novembre que sont tombées les averses les plus nombreuses $(1,7$ fois par an à Vialas, 1,5 à Valleraugue, 1,3 à Montpezat), mais aussi les plus importantes, avec une apparente prédilection pour les dates qui entourent le $1^{\text {er }}$ octobre. Ensuite, au cours des six mois de décembre à mai, le risque reste non négligeable et ne décroît que lentement avec une fréquence des averses encore voisine de la moitié de la fréquence d'automne. Pendant les trois mois de juin à août, le risque paraît beaucoup plus réduit $(0,2$ fois par an à Vialas et à Montpezat, 0,1 fois à Valleraugue). Il est cependant tombé $320 \mathrm{~mm}$ à Vialas le 21 juillet 1914 .

\section{$2^{\circ}$ Le phénomène n'est pas imprévisible.}

Nous décrirons ci-dessous les grandes lignes de l'évolution météorologique et essaierons d'en dégager quelques règles prévisionnelles.

\section{a) Evolutron de la situation météorologigue LES 29-30 SEPTEMBRE ET 3-4 OCTOBRE 1958:}

Succédant à une période de beau temps due à la présence de hautes pressions, le temps sur la France devient progressivement nuageux par l'ouest à partir du 27 au soir. La figure 7 représente les situations météorologiques au sol et en altitude $(700 \mathrm{mb})$ à $12 \mathrm{~h}$ TU, les 29,30 septembre et $1^{\mathrm{er}}$ octobre.

Le 29 septembre, la situation isobarique est caractérisée à 12 heures par une dépression au large de l'Irlande, et des hautes pressions sur l'Europe Centrale. Sur la face sud-est de la dépression, une perturbation donne un ciel nuageux à couvert par nuages moyens, généralement instables, du solfe de Gascogne à la mer du Nord.

Le fronl froid n'a pas encore abordé l'Espagne. On note par ailleurs la présence d'altocumulus en banc a Millau et Perpignan, en couche discontinue à Marseille, et des vents modérés à forts de secteur sud sur le Languedoc et le BasRhône.

A $700 \mathrm{mb}$, le contour reproduit sensiblement la carte sol. On remarquera cependant les points suivants:

1. Sur le nord-ouest de l'Espagne existe un fort gradient thermique à l'avant du front froid;

2. Une coupure existe sur le Maroc dans la ceinture de hautes pressions, permettant à des masses d'air tropical humide de pénétrer en Méditerranée.
Le 30 seplembre, la dépression est venuc se centrer sur l'Irlande. La zone de ciel couvert el pluvieux s'étend de la Méditerranée à l'est de la France, tandis que la moitié ouest est passéc dans l'air polaire avec averses de traîne.

Des vents forts de secteur sud persistent sur la basse vallée du Rhône.

A $700 \mathrm{mb}$, l'évolution a élé semblable à celle au sol. On notait :

1. L'accélération du flux de sud-sud-ouest qui dépasse $100 \mathrm{~km} / \mathrm{h}$ sur le sud-est de la France.

2. Le net accroissement du gradient thermique entre Bordeaux et Nîmes (le 29, températures: à Bordeaux +2 , à Nîmes +3 ; le 30 , Bordeaux - 2, Nimes +5 ), tandis que l'air reste relativement froid $(-1$ à Milan) sur le nord de l'Italie.

Le $1^{\text {cr }}$ octobre, l'air polaire atlantique a envahi completement la France, y donnant un temps de traine avec averses.

L’évolution, $d u 3$ au \& octobre, présente plusieurs points communs:

1. Courant chaud de sud à sud-ouest de l'A.F.N. au sud-est de la France, à l'avant d'une advection d'air polaire.

2. Vents forts de secteur sud à l'Aigoual, au Ventoux et à la Jungfrau.

3. Présence de nuages moyens sur le nord-est de l'Espagne et le Roussillon.

\section{b) Essai D'EXPlication DU PHÉNOMÈNE ET RECHERCHE D'ÉLÉMENTS PRÉVISIONNETS:}

La basse vallée du Rhône se présente comme un entonnoir très ouvert au sud et resserré au nord entre deux chaines de montagnes. Il en résulte une accélération du flux, mais aussi une ascendance forcée le long du relief, Ia masse d'air étant gênée dans son écoulement vers le nord. La langue d'air chaud canalisée par la vallée du Rhòne résiste au coincement entre les deux masses d'air froid, tandis que, sous l'influcnee des reliefs du Massif Central et des Pyrénées, des ondulations se forment sur le front froid atlantique. On peut penser en particulier que l'infiltration d'un paquet d'air froid par le setuil de Naurouze attire, par effet cyclonique, une contre-offensive de l'air méditerranéen du golfe du Lion aux Cévennes; contre-offensive dont le heurt avec le front froid principal est la cause de l'averse torrentielle. (2).

on peut donc retenir comme éléments prévi-

(2) Cette interprétation est due à M. Fontaine, ingénieur à la Météorologie Nationale. 
sionnels possibles, dans les 12 heures qui précèdent la pluie:

1. L'existence de vent de secteur sud modéré à fort $(30$ à $50 \mathrm{~km} / \mathrm{h})$ à la Jungfrau, au Ventoux et à l'Aigoual.

2. La présence de nuages moyens abondants au voisinage du golfe du Lion.

3. Un gradient thermique devenant positif douest en est sur le sud de la France.

4. Alimentation en air tropical en altitude.

Les points suivants : flux convergent de la Catalogne à la vallée du Rhône, présence d'air froid sur l'Italie, ondulations locales sur le front froid atlantique, representent des possibilités d'aggravation du phénomène.
L'averse du matin du 4 a été annoncée comme probable au début de l'après-midi du 3 par la Météorologie Nationale, cette fois-ci mise en éveil : un avis spécial radiodiffusé a probablement permis d'éviter de nouvelles pertes de vies humaines.

Des situations météorologiques dangereuses sont apparues au cours de l'hiver dernier. Les 20 décembre 1958, 20 janvier, 3 février, 3 mars 1959 , le risque a été signalé chaque fois avant les averses par les services météorologiques. Les hauteurs d'eau moyennes précipitées sur le versant oriental des Cévennes ont été à ces occasions respectivement de l'ordre de $300,80,50,150 \mathrm{~mm}$.

En résumé, à condition que la situation météorologique soit surveillée, le risque, sinon l'ordre de grandeur des précipitations, peut ètre signalé au moins 6 à 12 heures avant leur début.

\section{E R R A T A}

Corrections à apporter au texte de l'article de M. F. Ferrari : "Théorie du puits en écoulement non permanent », paru dans le $n^{\circ} B$ de 1958 :

Page $787,2^{\circ}$ colonne, équation (5), lire $\Phi_{2} \ldots \ldots$, au lieu de $\Phi$.....

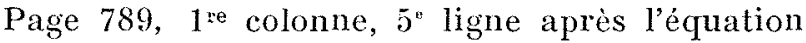
(11), lire « $\log »$ an lieu de $《 \log »$.

Page $790,1^{\text {re }}$ colonne, ligne 17 et dernière ligne, et $2^{\circ}$ colonne, lignes $5,8,10,14$, équation (17), 24 et 27 équation (18), lire «Log», au lieu de «log». 